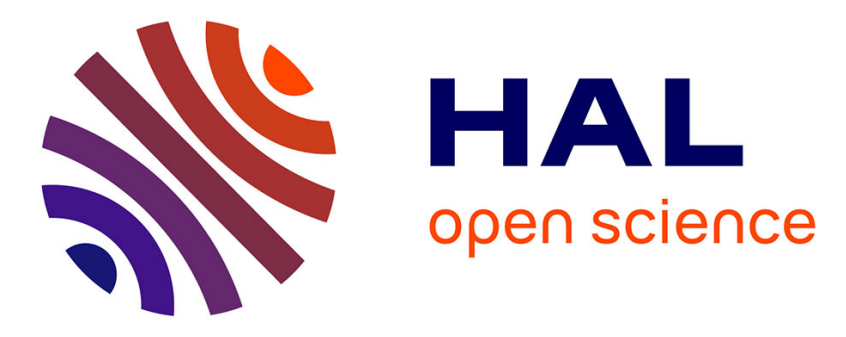

\title{
Analysis of fibrous elastic composites with nonuniform imperfect adhesion
}

\author{
Raul Guinovart-Diaz, Reinaldo Rodriguez-Ramos, Juan Carlos \\ López-Realpozo, Julian Bravo-Castillero, José Antonio Otero, Frederico J. \\ Sabina, Frédéric Lebon, Serge Dumont
}

\section{To cite this version:}

Raul Guinovart-Diaz, Reinaldo Rodriguez-Ramos, Juan Carlos López-Realpozo, Julian BravoCastillero, José Antonio Otero, et al.. Analysis of fibrous elastic composites with nonuniform imperfect adhesion. Acta Mechanica, 2016, 227 (1), pp.57-73. 10.1007/s00707-015-1428-x . hal-01293014

\section{HAL Id: hal-01293014 https://hal.science/hal-01293014}

Submitted on 20 May 2018

HAL is a multi-disciplinary open access archive for the deposit and dissemination of scientific research documents, whether they are published or not. The documents may come from teaching and research institutions in France or abroad, or from public or private research centers.
L'archive ouverte pluridisciplinaire $\mathbf{H A L}$, est destinée au dépôt et à la diffusion de documents scientifiques de niveau recherche, publiés ou non, émanant des établissements d'enseignement et de recherche français ou étrangers, des laboratoires publics ou privés. 


\title{
Analysis of fibrous elastic composites with nonuniform imperfect adhesion
}

\author{
R. Guinovart-Díaz · R. Rodríguez-Ramos • \\ J. C. López-Realpozo · J. Bravo-Castillero · J. A. Otero • \\ F. J. Sabina - F. Lebon · S. Dumont
}

\begin{abstract}
In most composites, the fiber-matrix adhesion is imperfect; the continuity conditions for stresses and displacements are not satisfied. In this contribution, effective elastic moduli are obtained by means of the asymptotic homogenization method (AHM), for three-phase fibrous composites (matrix/mesophase/fiber) with parallelogram periodic cell. Interaction between fiber and matrix is considered, and this is called the mesophase model where the nonuniform mesophase is studied. Besides, there is another type of
\end{abstract}

The co-authors JBC, RGD, JAO, JCLR and RRR would like to dedicate this work to Professor Sabina, F. J. in his anniversary.

R. Guinovart-Díaz · R. Rodríguez-Ramos $(\bowtie) \cdot J$. C. López-Realpozo · J. Bravo-Castillero

Facultad de Matemática y Computación, Universidad de La Habana, San Lázaro y L, Vedado,

Havana 4 CP 10400, Cuba

E-mail: reinaldo@matcom.uh.cu

R. Guinovart-Díaz

E-mail: guino@matcom.uh.cu

J. C. López-Realpozo

E-mail: jclrealpozo@matcom.uh.cu

J. Bravo-Castillero

E-mail: jbravo@matcom.uh.cu

\section{J. A. Otero}

Instituto Tecnológico y de Estudios Superiores de Monterrey,

Campus Estado de México, 52929 Atizapán de Zaragoza, México

E-mail: j.a.otero@itesm.mx

\section{F. J. Sabina}

Instituto de Investigaciones en Matemáticas Aplicadas y en Sistemas, Universidad Nacional

Autónoma de México, Delegación de Álvaro Obregón, Apartado Postal 20-726,

01000 México, D.F., Mexico

E-mail: fjs@mym.iimas.unam.mx

F. Lebon · S. Dumont

Laboratoire de Mécanique et d'Acoustique, CNRS, Centrale Marseille, Université Aix-Marseille,

31Chemin Joseph-Aiguier, 13402 Marseille Cedex 20, France

E-mail: lebon@lma.cnrs-mrs.fr

S. Dumont

Laboratoire Amiénois de Mathématiques Fondamentale et Appliquée CNRS UMR 7352,

Université de Picardie Jules Verne, 33, rue Saint Leu, 80039 Amiens, France

E-mail: serge.dumont@u-picardie.fr 
matrix-fiber contact which is called nonuniform spring imperfect contact. In this case, the contrast or jump of the displacements in the boundary of each phase is proportional to the corresponding component of the tension in the interface in terms of a parameter given by a certain function that depends on the position. The constituents of the composites exhibit transversely isotropic properties. A doubly periodic parallelogram array of cylindrical inclusions under longitudinal shear is considered. The three-phase model is validated by the Finite Element Method and the AHM both approaches applied to two-phase composites with nonuniform spring imperfect contact. Comparisons with theoretical and experimental results verified that the present model is efficient for the analysis of composites with presence of nonuniform imperfect interface and parallelogram cell. The effect of the nonuniform imperfection on the shear effective property is observed. The present method can provide benchmark results for other numerical and approximate methods.

\section{Introduction}

Composite materials containing microstructural constituents are very extensively used in industrial applications. However, defects induced due to manufacturing processes have been recognized as a general problem in this type of composites. These defects can be found in the fiber (i.e., fiber misalignment, irregular fiber distribution and broken fibers), in the matrix (i.e., voids and non-homogeneity) and/or in the interface regions (i.e., debonding, delimitations and imperfect interface) [1,2]. The presence of such defects affects the overall properties of composites and nonhomogeneous materials [3,4]. An imperfect interfacial bonding is a common defect in composites, which can significantly affect the mechanical properties of the material [5].

In most composites, the fiber-matrix adhesion is imperfect; the continuity conditions for stresses and displacement are not satisfied. Thus, various approaches have been used, in which the bond between the reinforcement and the matrix is modeled by an interphase with specified thickness [6,7]. Another approach consists in considering that the thickness and the elasticity coefficients of the bond are small parameters compared to those of the other components of the composite. Then, using matched asymptotic expansions, it can be shown that in that case, the behavior of the interphase can be accurately approximated by an interface with an appropriate contact law, where the jump in the displacements is proportional to the stress, which is supposed to be continuous (see, for example, [8-11]).

This kind of imperfect contact (spring type) in the modeling of composites was investigated by Benveniste and Miloh [12] among others and has been used, for instance, by Achenbach and Zhu [13] and Hashin [1416]. Some previous studies also reported the elastic effective coefficients for two-phase fibrous composites with rhombic array of periodic cells, both with perfect and with imperfect contact conditions, analytically and numerically [17-20].

In this work, a micromechanical analysis method is applied to a periodic composite with unidirectional fibers and parallelogram cells, in particular, rhombic periodic cells. The analytical expressions of the homogenized elastic properties are calculated for a two-phase composite with interphase. In this study, coated fibers or a thin mesophase is considered which often contains the contrast or jump of the displacements in the boundary of matrix and fibers [6]. The mesophase is assumed as a function that depends on the position (nonuniform imperfect adherence). The asymptotic homogenization method (AHM), for three-phase fibrous periodic composites with nonuniform mesophase and oblique cell, is used for the calculation of shear elastic effective coefficients. This contribution is an extension of previous works [21-23] where the imperfection on the interface is a constant function (uniform). The results in this paper are mainly focused on the impact of the arrangement of the fibers and the mechanical nonuniform imperfection at the interface on the stiffness properties. The novelty of the present work is that the imperfection of the interface in the composite with parallelogram cell is studied by two different models (three-phase and spring models) where the interface is in general nonuniform, which makes the composite more complex due to the presence of an heterogeneous interface. Moreover, both theoretical approaches are validated by the Finite Element Method.

\section{General considerations for three-phase composites: contact at the interface}

Let us consider a composite $\Omega$ of unidirectional periodic fibers embedded in a matrix as shown in Fig. 1 . The angle of the cell $\mu$ is assumed to remain constant so that a parallelogram cell with periods $w_{1}$ and $w_{2}$ can be defined. The periodicity of the microstructure determines the geometry of the periodic cell $S$. Thus, a three-phase periodic composite is considered here which consists of a parallelogram array of identical parallel circular concentric cylinders embedded in a homogeneous medium (Fig. 2). 

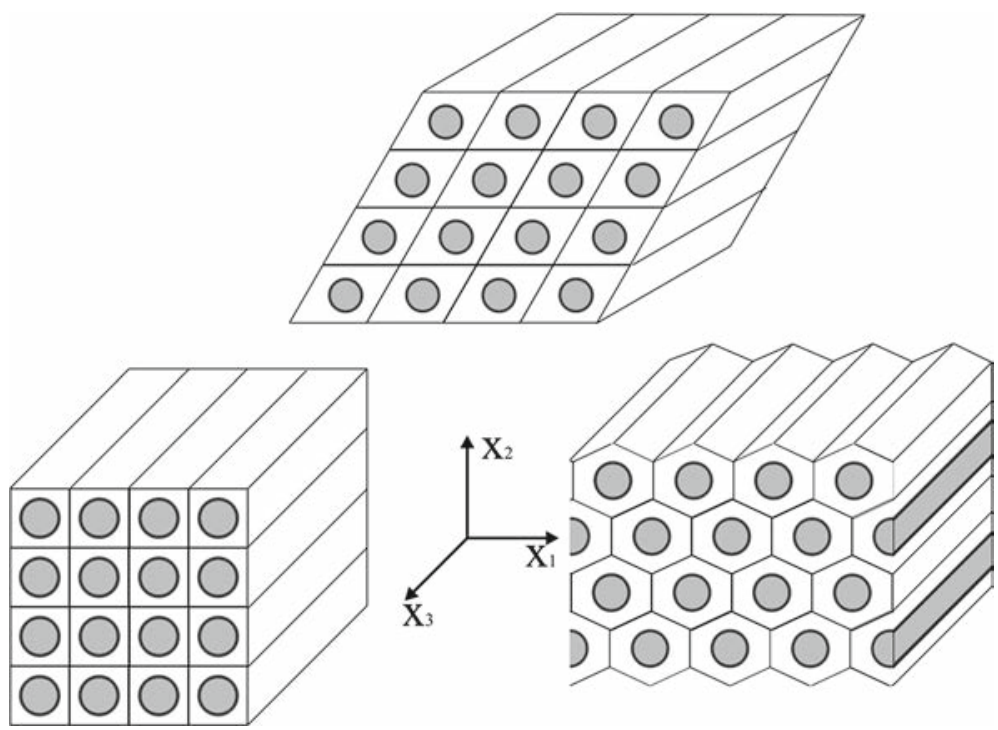

Fig. 1 Heterogeneous medium with periodic cell
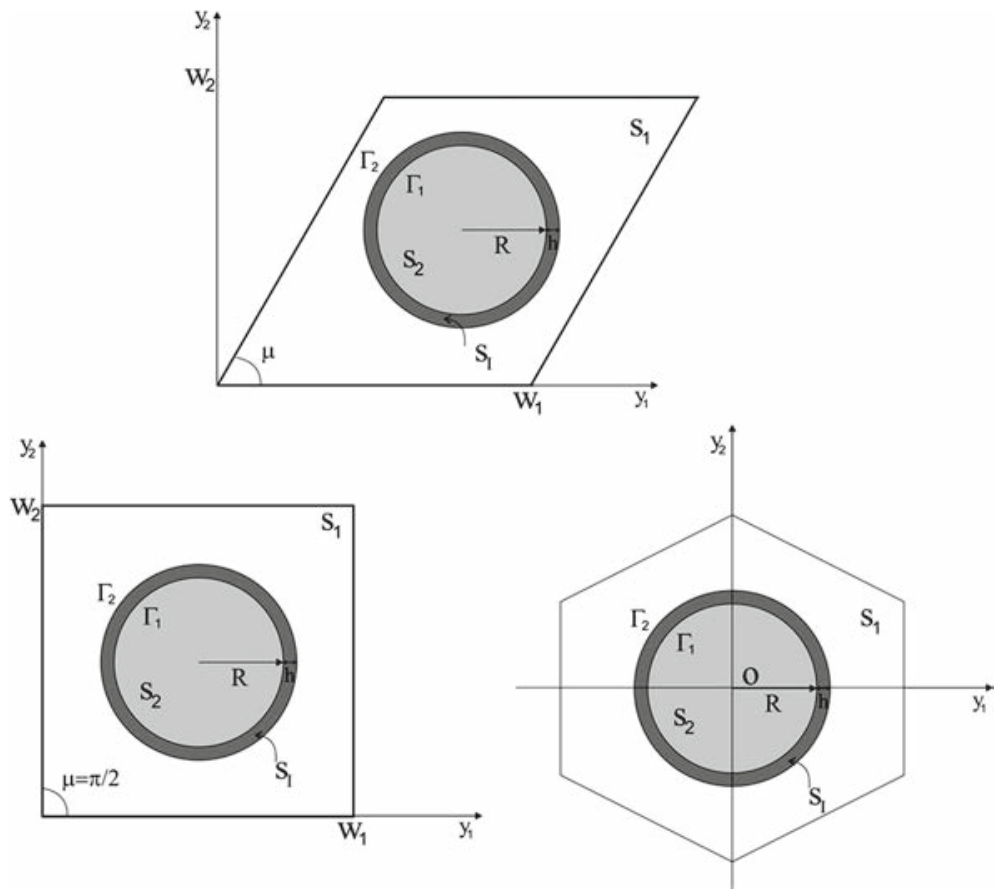

Fig. 2 The parallelogram, square and hexagonal cells showing the domains $S_{1}, S_{I}$ and $S_{2}$ occupied by the matrix, mesophase and fiber materials; $\Gamma_{2}, \Gamma_{1}$ are the common interfaces

As a unidirectional fibrous composite, it is assumed that the microstructure of the composite along the third direction (perpendicular to the plane of cross section) remains constant. The central fibers are all assumed straight and of circular cross section with radius $R$, and the mesophase has a thickness $h$ (see Fig. 2). The material properties of each phase belong to the crystal symmetry class $6 \mathrm{~mm}$, where the axes of material and geometric symmetry are parallel. The composite is not well bonded at the contact between the matrix and the fiber. Therefore, imperfect contact conditions at the interface $\Gamma^{\varepsilon}$ are considered, where $\varepsilon=l / L$ is a small geometrical parameter relating the distance $l$ between the centers of two neighboring cylinders and the diameter $L$ of the composite.

A family of boundary value problems depending on $\varepsilon$ for the linear elastic heterogeneous media can be formulated in general form for a three-phase composite as follows: 


$$
\begin{aligned}
& \nabla \cdot\left(\mathbf{C}^{\varepsilon}: \nabla \mathbf{u}^{\varepsilon}\right)=0, \quad \mathbf{x} \in \Omega, \\
& \llbracket \mathbf{T}\left(\mathbf{u}^{\varepsilon}\right) \rrbracket=\mathbf{0}, \quad \mathbf{x} \in \Gamma^{\varepsilon}, \\
& \llbracket \mathbf{u}^{\varepsilon} \rrbracket=0, \quad \mathbf{x} \in \Gamma^{\varepsilon}, \\
& \mathbf{u}=\mathbf{u}_{0} \text { on } \partial \Omega,
\end{aligned}
$$

where $\mathbf{C}^{\varepsilon}(\mathbf{x})=\mathbf{C}(\mathbf{y})$ is the $S$-periodic stiffness elastic tensor and $\mathbf{y}=\mathbf{x} / \varepsilon$ is the local variable. Moreover, $\nabla$ is the gradient operator and $\mathbf{u}^{\varepsilon}=\left(u_{1}^{\varepsilon}, u_{2}^{\varepsilon}, u_{3}^{\varepsilon}\right)$ the mechanical displacement vector referring to the Cartesian coordinate system. $\mathbf{u}_{0}$ is a prescribed displacement at the boundary $\partial \Omega$. T is the traction vector, and $\llbracket f \rrbracket_{\gamma}(\gamma=$ $1,2)$ indicates the jump in the quantity at the common interface $\Gamma^{\varepsilon}$ between the matrix and the mesophase, and the mesophase with the fiber, i.e, $\llbracket f \rrbracket_{1}=f^{(1)}-f^{(I)}, \llbracket f \rrbracket_{2}=f^{(I)}-f^{(2)}$.

Since a periodic composite with nonuniform interphase is studied, three distinct phases, occupying $S_{1}$, $S_{I}$ and $S_{2}$ (Fig. 2), are assumed. The region $S_{I}$ characterizes the non-perfect contact along the interfaces $\Gamma_{1}=\left\{z=R \mathrm{e}^{i \theta}, 0 \leq \theta \leq 2 \pi\right\}$ and $\Gamma_{2}=\left\{z=(R+h) \mathrm{e}^{i \theta}, 0 \leq \theta \leq 2 \pi\right\}$ of each cylinder of radius $R$ and $R_{1}=R+h$, respectively, where $h$ denotes the thickness of the mesophase $S_{I}$. The effect of mechanical imperfection in the effective properties of the composite is incorporated through the presence of the mesophase $S_{I}$. In the forthcoming sections, some summary ideas of the antiplane problem and a brief comment about the asymptotic homogenization method (AHM) are given.

\subsection{Asymptotic homogenization method (AHM) for the antiplane problems ${ }_{\alpha 3} \mathrm{~L}$}

It is well known that the problem (1)-(4) is decoupled into two independent systems for unidirectional fibrous composites with components of transversely isotropy symmetry. One of them involves $u_{1}^{\varepsilon}, u_{2}^{\varepsilon}$, i.e., it is a state of in-plane mechanical deformation field. The other state, which is of particular interest in this work, is characterized by an out-of-plane mechanical displacement $u_{3}^{\varepsilon}$ which is a function of the variables $x_{1}$ and $x_{2}$. The main aim of this section is the determination of effective properties in three-phase composites for the out of plane using the homogenization method, considering that the mesophase $S_{I}$ is not a homogeneous medium with its properties being functions of the angle $\theta$ with vertices in the center of the fiber (Fig. 3). In this case, the relevant constitutive relations are

$$
\sigma_{13}=C_{1313} u_{3,1}, \quad \sigma_{23}=C_{2323} u_{3,2},
$$

where $\sigma_{13}$ and $\sigma_{23}$ are the components of out-of-plane mechanical stress, $C_{1313}$ and $C_{2323}$ are the elastic moduli, and the comma denotes partial differentiation.

Here, we are interested to consider the antiplane case, and the BVP (1)-(4) is transformed into the problem

$$
\begin{gathered}
\left(C_{3 \eta 3 \beta}(\mathbf{y}) u_{3, \beta}\right)_{, \eta}=0, \text { in } \Omega, \\
\llbracket C_{1313}^{\varepsilon}\left(u_{3,1}^{\varepsilon} n_{1}+u_{3,2}^{\varepsilon} n_{2}\right) \rrbracket=0, \text { on } \Gamma^{\varepsilon}, \\
\llbracket u_{3}^{\varepsilon} \rrbracket=0, \text { on } \Gamma^{\varepsilon}, \\
u_{3}^{\varepsilon}=u_{0}, \quad \text { on } \partial \Omega,
\end{gathered}
$$

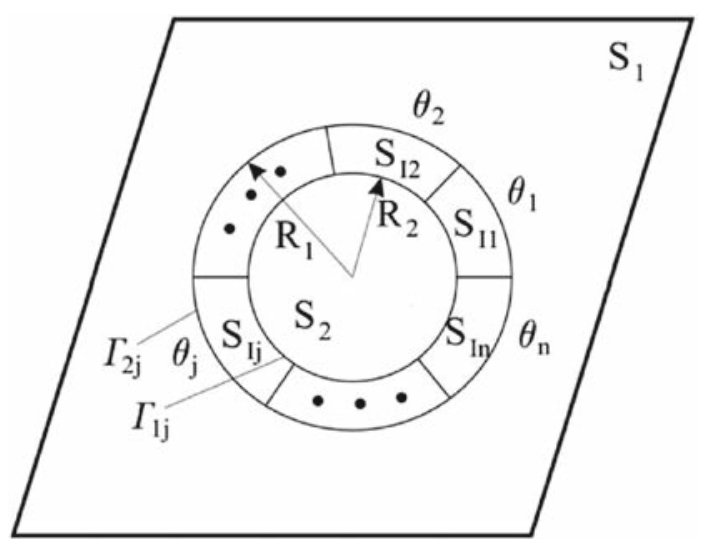

Fig. 3 Three-phase composite with nonuniform interface 
where the Greek indices run from 1 to 2 and $\Gamma^{\varepsilon}=\Gamma_{1} \cup \Gamma_{2}$. The usual convention of index summation is used.

Equation (6) has $S$-periodic coefficients and they are rapidly oscillating (see, Fig. 1). In order to obtain the homogenized problem and the corresponding effective coefficients, the solution of (6)-(9) is sought using the method of two scales by the ansatz:

$$
u_{3}^{\varepsilon}(\mathbf{x})=v_{0}(\mathbf{x})+\varepsilon v_{1}(\mathbf{x}, \mathbf{y})+O\left(\varepsilon^{2}\right), \quad \mathbf{x}=\left(x_{1}, x_{2}\right), \quad \mathbf{y}=\left(y_{1}, y_{2}\right),
$$

with $v_{1}$ being an $S$-periodic function with respect to $\mathbf{y}$. Substituting (10) into (6), applying the chain rule considering that $\mathbf{x}$ and $\mathbf{y}$ are independent and equating the terms of orders $\varepsilon^{-1}, \varepsilon^{0}$ to zero, one can obtain that $v_{1}(\mathbf{x}, \mathbf{y})={ }_{\alpha 3} N(\mathbf{y}) v_{0, \alpha}(\mathbf{x})$, where $v_{0}(\mathbf{x})$ is the solution of the homogenized equation $C_{3 \alpha 3 \beta}^{*} v_{0, \alpha \beta}=0$, where $C_{3 \alpha 3 \beta}^{*}=\left\langle C_{3 \alpha 3 \beta}(\mathbf{y})+C_{3 \alpha 3 \eta}(\mathbf{y})_{\alpha 3} N_{, \eta}(\mathbf{y})\right\rangle$ are the so-called effective coefficients and ${ }_{\alpha 3} N(\mathbf{y})$ is an $S$-periodic harmonic function of the $\alpha_{33} L$ local problem. The comma will also be used to denote a derivative with respect to the local variable. The angular brackets define the volume average per unit length over the unit cell, that is, $\langle F\rangle=\frac{1}{V} \int_{S} F(y) \mathrm{d} y$. The index $\alpha=1,2$ denotes two different problems over the periodic cell which are to be solved. It is a well-known derivation whose details can be found elsewhere and is omitted here (see, for instance, $[24,25])$.

The local problems are referred to as ${ }_{13} L$ and ${ }_{23} L$ with solutions ${ }_{13} N(\mathbf{y})$ and ${ }_{23} N(\mathbf{y})$, respectively. The solution of such local problems on the periodic cell described in Fig. 2, taking into account the perfect contact conditions (7) and (8), is one of the main objectives of the present work to obtain the overall properties $C_{55}^{*}, C_{54}^{*}, C_{45}^{*}$ and $C_{44}^{*}$ with the short index notation. The alternative form is found as follows:

$$
\begin{aligned}
& C_{55}^{*}=p_{1} V_{1}+p_{I} V_{I}+p_{2} V_{2}+\left\langle p_{13} N_{, 1}\right\rangle, \\
& C_{54}^{*}=\left\langle p_{13} N_{, 2}\right\rangle, \quad C_{45}^{*}=\left\langle p_{23} N_{, 1}\right\rangle, \\
& C_{44}^{*}=p_{1} V_{1}+p_{I} V_{I}+p_{2} V_{2}+\left\langle p_{23} N_{, 2}\right\rangle,
\end{aligned}
$$

where $p_{\gamma}=C_{1313}^{(\gamma)}$ with $(\gamma=1, I, 2)$ are the shear moduli of the matrix, mesophase and fibers, respectively. $V_{2}=\pi R^{2} / V, V_{I}=\pi\left(2 h R+h^{2}\right) / V$ and $V_{1}=1-V_{I}-V_{2}$ are the percentages of concentrations of fiber, mesophase and matrix, respectively, and $V=\left|w_{1}\right|\left|w_{2}\right| \sin \mu$ is the area of periodic cell.

The mathematical statement for the two local problems $\alpha_{3} L(\alpha=1,2)$ in the case of three-phase composites consist of finding doubly periodic functions ${ }_{\alpha 3} N={ }_{\alpha 3} N^{(\gamma)}(\mathbf{y})$ if $\mathbf{y} \in S_{\gamma}$, that satisfy the following Laplace equations with the contact condition in each phase $(\gamma=1, I, 2)$ :

$$
\nabla_{\alpha 3}^{2} N^{(\gamma)}=0 \text { in } S_{\gamma}
$$

where the continuity condition for the stress and displacement at the interfaces $\Gamma_{1}$ and $\Gamma_{2}$ depends on the angle $\theta$ of the point $z=\mathrm{re}^{i \theta} \in S_{I}$, which describes a particular type of imperfect contact between the matrix and fiber with nonhomogeneous mesophase. The interface $\Gamma^{\varepsilon}=\Gamma_{1} \cup \Gamma_{2}$ by the continuity of displacement, therefore, yields

$$
\llbracket{ }_{\alpha 3} N \rrbracket=0 \quad \text { on } \Gamma^{\varepsilon}=\Gamma_{1} \cup \Gamma_{2},
$$

and the continuity condition for the stress

$$
\llbracket p_{\gamma}\left(\alpha_{3} N_{, 1}^{(\gamma)} n_{1}+{ }_{\alpha 3} N_{, 2}^{(\gamma)} n_{2}\right) \rrbracket=-\llbracket p_{\gamma} \rrbracket n_{\alpha} \quad \text { on } \Gamma^{\varepsilon}=\Gamma_{1} \cup \Gamma_{2},
$$

and the condition for uniqueness

$$
\left\langle{ }_{\alpha 3} N\right\rangle=0 \text { in } S=S_{1} \cup S_{I} \cup S_{2} .
$$

The outward unit normal vector to the interface $\Gamma$ is $\mathbf{n}=\left(n_{1}, n_{2}\right)$. The pre-index used in (14)-(17) for the local function will be omitted for simplicity. The problem (14)-(17) should be converted into dimensionless problems making the appropriate change. Indeed, defining the dimensionless variable $\xi=y / l$ (this is not a small parameter!), then $N_{, \beta}^{(\gamma)}=u_{, \beta}^{(\gamma)}$ and $N_{, \beta \beta}^{(\gamma)}=u_{, \beta \beta}^{(\gamma)} / l$ where $u^{(\gamma)} \equiv N^{(\gamma)} / l$ and the derivative $u_{, \beta}$ is with respect to the variable $\xi_{\beta}$. $R_{0}$ is the true radius of the fibers in the composite. Also the dimensionless parameter $R=R_{0} / l$ is introduced. 
2.2 Solution of local problems ${ }_{\alpha 3} L(\alpha=1,2)$ for three-phase composites with nonuniform imperfect mesophase

The well-developed theory of analytical functions in Muskhelishvili [26] can be applied to solve the problem (14)-(17). Thus, it is necessary to solve the local problems ${ }_{\alpha 3} L$. Doubly periodic harmonic functions in the matrix $(\gamma=1)$, mesophase $(\gamma=I)$ and fiber $(\gamma=2)$ region are to be found for the ${ }_{\alpha 3} L$ local problems in terms of harmonic functions $\varphi_{\gamma}(z)$ analogous to Lopez-Realpozo et al. [23],

$$
u^{(\gamma)}=\operatorname{Re}\left\{\varphi_{\gamma}(z)\right\}
$$

Since the composite exhibits a nonuniform mesophase between the matrix and fiber, we consider a partition of the cell $S_{\gamma}=\bigcup_{j=1}^{n} S_{\gamma j}$, where $S_{\gamma j}(\theta)=\left\{z=\mathrm{re}^{i \theta}: z \in S_{\gamma}, \theta_{j-1}<\theta<\theta_{j}\right\}$. This partition divides the interfaces $\Gamma_{\beta}=\bigcup_{j=1}^{n} \Gamma_{\beta j}, \Gamma_{\beta i} \cap \Gamma_{\beta j}=\varnothing, i \neq j, \beta=1$, 2, into $n$ arcs. Over the mesophase $S_{I}$, the material property $p_{I}$ is a piecewise constant function $p_{I}(\theta)=p_{I j}, \theta_{j-1}<\theta<\theta_{j}$ with $j=1, \ldots, n$, $\theta_{0} \equiv 0, \theta_{n} \equiv 2 \pi$ and $\theta_{j}$ denotes the angle of each arc (Fig. 3).

In each region $S_{\gamma j}$, we consider

$$
\begin{aligned}
\varphi_{1 j} & =\frac{a_{0 j} z}{R_{1}}+\sum_{k=1}^{\infty} o\left(\frac{\zeta^{(k-1)}\left(z / R_{1}\right)}{(k-1) !}\right) a_{k j}, \\
\phi_{I j}(z) & =\sum_{p=1}^{\infty}{ }^{o} b_{p j}\left(\frac{R_{1}}{z}\right)^{p}+\sum_{p=1}^{\infty}{ }^{o} b_{-p j}\left(\frac{z}{R}\right)^{p}, \\
\varphi_{2 j}(z) & =\sum_{p=1}^{\infty}{ }^{o}\left(\frac{z}{R}\right)^{p} c_{p j} .
\end{aligned}
$$

$\zeta(z)$ is the Weierstrass quasi-periodic function, which is defined as $\zeta(z)=\frac{1}{z}+\sum_{m, n}^{\infty}{ }^{\prime}\left(\frac{1}{z-P_{n m}}+\frac{1}{P_{n m}}+\frac{z}{P_{n m}^{2}}\right)$, $P_{n m}=n w_{1}+m w_{2}$, for $m, n \in Z$, and the prime over the summation symbol means that the pair $(m, n)=$ $(0,0)$ is excluded. The Laurent's expansion of function $\varphi_{1}^{j}(z)$ is given by the following expression:

$$
\varphi_{1 j}(z)=\frac{z}{R} a_{0 j}+\sum_{p=1}^{\infty} o\left(\frac{R_{1}}{z}\right)^{p} a_{p j}-\sum_{p=1}^{\infty} o \sum_{k=1}^{\infty} o\left(\frac{z}{R_{1}}\right)^{p} \sqrt{\frac{k}{p}} w_{k p} a_{k j},
$$

where $w_{k p}=\frac{(k+p-1) !}{(k-1) !(p-1) !} \frac{S_{k+p} R_{1}^{k+p}}{\sqrt{k p}}, S_{k+p}=\sum_{m, n}\left(m w_{1}+n w_{2}\right)^{-(k+p)}, m^{2}+n^{2} \neq 0, \quad k+p \geq 2, S_{2}=0$.

The constants $a_{0 j}, a_{p j}, b_{p j}, b_{-p j}, c_{p j}$ and $z=\xi_{1}+i \xi_{2}$ are complex numbers; the overbar denotes the complex conjugate and the superscript "o" on the summation indicates that the sum is carried out only over odd indices. $w_{1}, w_{2}$ are the periods and $a_{0 j}=-R_{1}^{2} H_{1} \bar{a}_{1 j}-R_{1}^{2} H_{2} a_{1 j}$, where $H_{1}=\frac{\bar{\delta}_{1} \bar{w}_{2}-\bar{\delta}_{2} \bar{w}_{1}}{w_{1} \bar{w}_{2}-w_{2} \bar{w}_{1}}, H_{2}=\frac{\delta_{1} \bar{w}_{2}-\delta_{2} \bar{w}_{1}}{w_{1} \bar{w}_{2}-w_{2} \bar{w}_{1}}$, with $\delta_{i}=\zeta\left(z+w_{i}\right)-\zeta(z)$.

Replacing (18)-(22) into (15)-(16) and performing some algebraic manipulations, the following system of equations is obtained in order to find the complex unknown coefficients $a_{k j}$ for each arc by the system:

$$
\bar{a}_{p j}+\chi_{1 j} R_{1}^{2} H_{1} \delta_{1 p} \bar{a}_{1 j}+\chi_{1 j} R_{1}^{2} H_{2} \delta_{1 p} a_{1 j}+\chi_{p j} \sum_{k=1}^{\infty}{ }^{o} w_{k p} a_{p j}=R_{1} \chi_{1 j} \delta_{1 p}\left(\delta_{1 \alpha}-i \delta_{2 \alpha}\right),
$$

where $\delta_{k p}$ is the Kronecker delta, $\chi_{p j}=\frac{\left(V_{2}+V_{I}\right)^{p}\left(\kappa_{1 j}+\kappa_{2}\right)\left(1-\kappa_{1 j}\right)+V_{2}^{p}\left(\kappa_{1 j}-\kappa_{2}\right)\left(1+\kappa_{1 j}\right)}{\left(V_{2}+V_{I}\right)^{p}\left(\kappa_{1 j}+\kappa_{2}\right)\left(1+\kappa_{1 j}\right)+V_{2}^{p}\left(\kappa_{1 j}-\kappa_{2}\right)\left(1-\kappa_{1 j}\right)}, \kappa_{2}=p_{2} / p_{1}$ and $\kappa_{1 j}=$ $p_{I j} / p_{1}$ are the components of shear ratios for each region $S_{I j}(\theta)$ of the mesophase with the shear property of the matrix. 
2.3 Effective properties for three-phase composites under nonuniform mesophase

The Eqs. (11)-(13) are easily transformed applying Green's theorem to the area integrals. Replacing $N^{(\gamma)}=$ $u^{(\gamma)} / l, \mathrm{~d} y_{i}=l \mathrm{~d} \xi_{i}$ and neglecting the line integral over the radial path in $S_{I}$ because its thickness $h \ll 1$, the effective coefficients $C_{55}^{*}, C_{54}^{*}, C_{45}^{*}$ and $C_{44}^{*}$ are connected by the following relations:

$$
\begin{aligned}
C_{55}^{*}-i C_{54}^{*}=\langle p\rangle-\sum_{j=1}^{n} \frac{p_{1}-p_{I j}}{V}\left(\int_{\Gamma_{1 j}} u^{(1)} \mathrm{d} x_{2}+i \int_{\Gamma_{1 j}} u^{(1)} \mathrm{d} x_{1}\right) \\
+\frac{p_{I j}-p_{2}}{V}\left(\int_{\Gamma_{2 j}} u^{(2)} \mathrm{d} x_{2}+i \int_{\Gamma_{2 j}} u^{(2)} \mathrm{d} x_{1}\right), \\
C_{45}^{*}-i C_{44}^{*}=-i\langle p\rangle-\sum_{j=1}^{n} \frac{p_{1}-p_{I j}}{V}\left(\int_{\Gamma_{1 j}} u^{(1)} \mathrm{d} x_{2}+i \int_{\Gamma_{1 j}} u^{(1)} \mathrm{d} x_{1}\right) \\
+\frac{p_{I j}-p_{2}}{V}\left(\int_{\Gamma_{2 j}} u^{(2)} d x_{2}+i \int_{\Gamma_{2 j}} u^{(2)} \mathrm{d} x_{1}\right)
\end{aligned}
$$

where $\langle p\rangle=p_{1} V_{1}+p_{2} V_{2}+\sum_{j=1}^{n} p_{I j} V_{I j}$ is the Voigt average, $V_{I}=\sum_{j=1}^{n} p_{I j} V_{I j}$ is the volume fraction of the mesophase and $V_{I j}$ denotes the volume fraction of the region $S_{I j}$. The volume fractions of matrix, fiber and the parallelogram periodic cell $\left(V_{1}, V_{2}\right.$ and $\left.V\right)$ were defined previously. In $(24), u^{(\gamma)}$ is the solution of the local problem ${ }_{13} L$, whereas in $(25) u^{(\gamma)}$ is the solution of ${ }_{23} L$. Taking into account (18)-(22), the analytical formulae for effective properties are obtained from (24) and (25) depending only on the unknown $a_{1 j}$ as follows:

$$
\begin{aligned}
& C_{55}^{*}-i C_{54}^{*}=\langle p\rangle-\sum_{j=1}^{n}{ }_{13} Q_{1 j}\left(\theta_{j+1}-\theta_{j}\right)-\sum_{j=1}^{n}{ }_{13} \bar{Q}_{1 j} e^{-i\left(\theta_{j+1}+\theta_{j}\right)} \operatorname{sen}\left(\theta_{j+1}-\theta_{j}\right) \\
& -2 \sum_{j=1}^{n}\left[\begin{array}{c}
\sum_{p=3}^{\infty} 13 Q_{p j} \frac{e^{\frac{i(p-1)\left(\theta_{j+1}+\theta_{j}\right)}{2}}}{p-1} \operatorname{sen} \frac{(p-1)\left(\theta_{j+1}-\theta_{j}\right)}{2} \\
+\sum_{p=3}^{\infty} 13 \bar{Q}_{p j} \frac{e^{\frac{-i(p+1)\left(\theta_{j+1}+\theta_{j}\right)}{2}}}{p+1} \operatorname{sen} \frac{(p+1)\left(\theta_{j+1}-\theta_{j}\right)}{2}
\end{array}\right], \\
& C_{45}^{*}-i C_{44}^{*}=-i\langle p\rangle-\sum_{j=1}^{n}{ }_{23} Q_{1 j}\left(\theta_{j+1}-\theta_{j}\right)-\sum_{j=1}^{n}{ }_{23} \bar{Q}_{1 j} e^{-i\left(\theta_{j+1}+\theta_{j}\right)} \operatorname{sen}\left(\theta_{j+1}-\theta_{j}\right) \\
& -2 \sum_{j=1}^{n}\left[\begin{array}{c}
\sum_{p=3}^{\infty} 23 Q_{p j} \frac{e^{\frac{i(p-1)\left(\theta_{j+1}+\theta_{j}\right)}{2}}}{p-1} \operatorname{sen} \frac{(p-1)\left(\theta_{j+1}-\theta_{j}\right)}{2} \\
+\sum_{p=3}^{\infty} 23 \bar{Q}_{p j} \frac{e^{\frac{-i(p+1)\left(\theta_{j+1}+\theta_{j}\right)}{2}}}{p+1} \operatorname{sen} \frac{(p+1)\left(\theta_{j+1}-\theta_{j}\right)}{2}
\end{array}\right],
\end{aligned}
$$

where

$$
\begin{aligned}
{ }_{\alpha 3} Q_{p j}= & {\left[\left(p_{1}-p_{I j}\right) R_{1}-\frac{\left(p_{2}-p_{I j}\right) \kappa_{1 j} R}{A_{p j}}\right] \frac{\left(\chi_{p j}+1\right) \bar{a}_{p}^{j}-R_{1} \chi_{1 j}\left(\delta_{1 \alpha}-i \delta_{2 \alpha}\right) \delta_{1 p}}{2 V \chi_{p j}} } \\
& -\frac{\left(p_{2}-p_{I j}\right)}{2 A_{1 j} V}\left(\frac{R^{2}-R_{1}^{2}}{R_{1} R}\right) \frac{\left(\kappa_{2}-\kappa_{1 j}\right)}{2} R^{2}\left(\delta_{1 \alpha}-i \delta_{2 \alpha}\right) \delta_{1 p}, \\
A_{p j}= & \frac{\left(\kappa_{1 j}+\kappa_{2}\right) R_{1}^{2 p}+\left(\kappa_{1 j}-\kappa_{2}\right) R^{2 p}}{2 R_{1}^{p} R^{p}} .
\end{aligned}
$$


The coefficients (26) and (27) represent the effective axial coefficients of a reinforced three-phase composite with nonuniform mesophase $S_{I}$. The analytical expressions of the effective properties (26) and (27) are functions of the properties and volume fractions of constituents of a composite with periodic cell $w_{1}, w_{2}$. As a limit case, the two-phase composite with perfect contact on interface $\Gamma$ reported in Guinovart-Díaz et al. [22] is obtained from (26) and (27) by taking the parameters $\kappa_{1 j}=p_{I j} / p_{1} \rightarrow \infty$. The complete separation is obtained when $\kappa_{1 j}=p_{I j} / p_{1} \rightarrow 0$.

\section{Local problems ${ }_{\alpha 3} \tilde{L}(\alpha=1,2)$ and effective properties for two-phase composites under nonuniform spring imperfect contact}

The statement and the solution of the local problems as well as the effective coefficients for nonuniform imperfect spring model are calculated in an analogous way to the three-phase model reported in the previous section, and the principal details can be found in López-Realpozo et al (2014). Therefore, in this section we shall not repeat the same procedure and only some fundamental ideas will be explained, and the closed-form expressions of the effective coefficients will be written.

Now, the family of boundary value problems depending on $\varepsilon$ for the linear elastic heterogeneous media can be formulated in general form as follows:

$$
\begin{aligned}
& \nabla \cdot\left(\mathbf{C}^{\varepsilon}: \nabla \mathbf{w}^{\varepsilon}\right)=0, \quad \mathbf{x} \in \Omega, \\
& \llbracket \mathbf{T}\left(\mathbf{w}^{\varepsilon}\right) \rrbracket=\mathbf{0}, \quad \mathbf{x} \in \Gamma^{\varepsilon}, \\
& \mathbf{T}^{(\gamma)}\left(\mathbf{w}^{\varepsilon}\right)=(-1)^{\gamma+1} \mathbf{K} \llbracket \mathbf{w}^{\varepsilon} \rrbracket, \quad \mathbf{x} \in \Gamma^{\varepsilon}, \\
& \mathbf{w}^{\varepsilon}(\mathbf{x})=\mathbf{A u}_{0}(\mathbf{x}), \quad \mathbf{x} \in \partial \Omega,
\end{aligned}
$$

where $\mathbf{C}^{\varepsilon}(\mathbf{x})=\mathbf{C}(\mathbf{y})$ is the $S$-periodic stiffness elastic tensor and $\mathbf{y}=\mathbf{x} / \varepsilon$ is the local variable. Moreover, $\mathbf{w}^{\varepsilon}=\mathbf{A} \mathbf{u}^{\varepsilon}, \mathbf{A}$ is a rotation matrix, the spring stiffness diagonal matrix is given by $\mathbf{K}$, the displacement and the traction vectors are denoted by $\mathbf{w}$ and $\mathbf{T}$, respectively, where

$$
\mathbf{K}=\left(\begin{array}{lll}
\widetilde{K}_{n} & 0 & 0 \\
0 & \widetilde{K}_{t} & 0 \\
0 & 0 & \widetilde{K}_{s}
\end{array}\right), \quad \mathbf{w}=\left(\begin{array}{c}
w_{n} \\
w_{t} \\
w_{s}
\end{array}\right), \quad \mathbf{T}=\left(\begin{array}{c}
T_{n} \\
T_{t} \\
T_{s}
\end{array}\right) .
$$

The corresponding tractions remain continuous (29), while the effect of mechanical imperfection is incorporated through the mechanical displacements jumps across the interface (30). The spring constants $\mathbf{K}$ are the measures for the magnitude of the associated continuities, and they are a function of the position at the interface which is called proportional interface parameter [27]. The vanishing value of $\tilde{K}_{n}$ and $\tilde{K}_{t}, \tilde{K}_{s}$ corresponds to pure debonding (normal perfect debonding), in-plane pure sliding and out-of-plane pure sliding, respectively. The status of the mechanical bonding is completely determined by appropriate values of these constants. For large enough values of the constants, the perfect bonding interface is achieved.; $w_{n}, w_{t}, w_{s}$ are the normal and the two tangential components of the rotated mechanical displacement vector $\mathbf{w}^{\varepsilon} ; T_{n}, T_{t}, T_{s}$ are the normal and tangential components of the traction vector $\mathbf{T}\left(T_{i}=\sigma_{i j} n_{j}\right), \mathbf{n}$ is the outward unit normal on $\Gamma$.

Here also we are interested to consider the antiplane case, and then, dimensionless local problems ${ }_{\alpha 3} \tilde{L}(\alpha=$ $1,2)$ related to (28)-(31) over the periodic cell $S$ are written as follows:

Laplace equation:

$$
\nabla^{2} u^{(\gamma)}=0, \text { in } S_{\gamma}
$$

imperfect contact condition:

$$
\frac{p_{\gamma}\left(u_{, 1}^{(\gamma)} n_{1}+u_{, 2}^{(\gamma)} n_{2}\right)+p_{\gamma} n_{\alpha}}{p_{1}}=\frac{K_{s}(\theta)}{R} \llbracket u \rrbracket, \text { on } \Gamma,
$$

continuity condition for the stress:

$$
\llbracket \frac{p_{\gamma}\left(u_{, 1}^{(\gamma)} n_{1}+u_{, 2}^{(\gamma)} n_{2}\right)}{p_{1}} \rrbracket=-(1-\kappa) n_{\alpha}, \text { on } \Gamma,
$$

uniqueness condition:

$$
\langle u\rangle=0, \text { in } S=S_{1} \cup S_{2},
$$



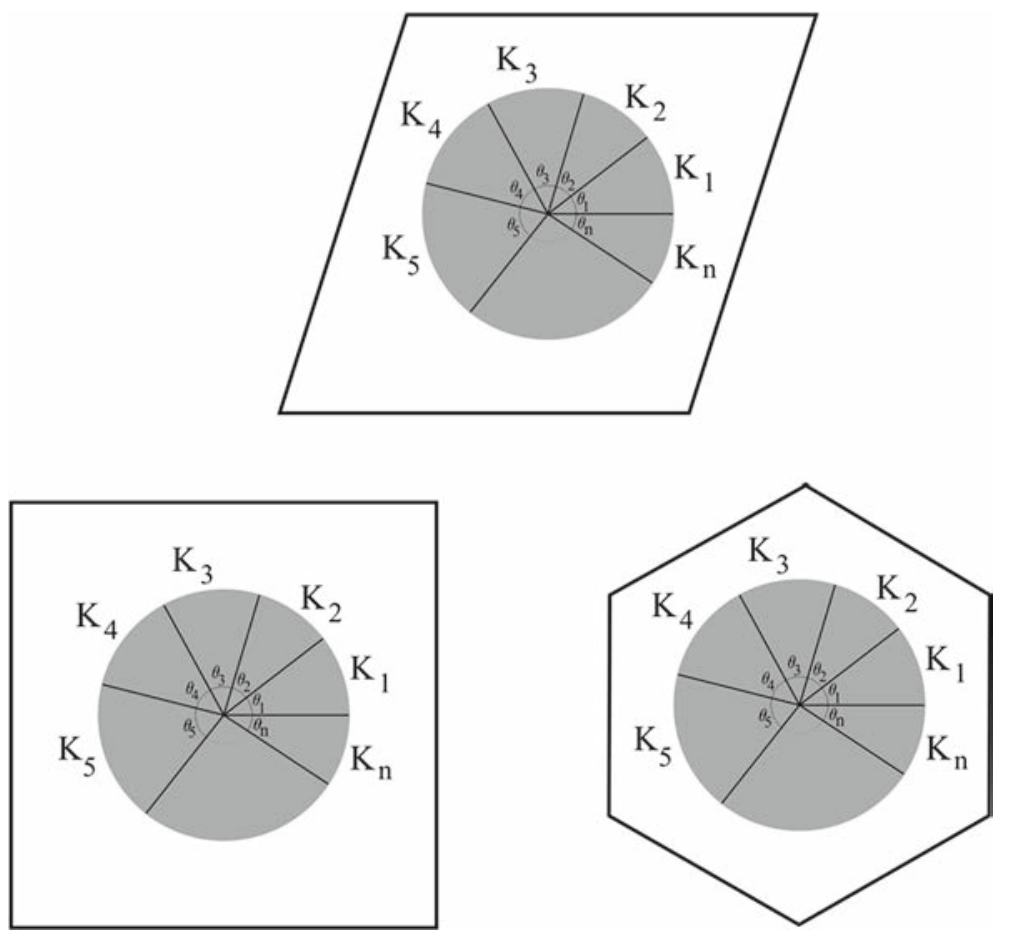

Fig. 4 Partition of the interface in $n$ arcs for the spring model

where $\kappa=p_{2} / p_{1}$ and the superscripts $\gamma, \gamma=1,2$ denote the matrix and fiber, respectively. The interface parameters in (32) are replaced by dimensionless constants. Consequently, $\widetilde{K}_{s}=K_{S}(\theta) p_{1} / R$, where $K_{S}(\theta)$ is a dimensionless function that depends on the angle $\theta$ (Fig. 4).

The analytical formulae for effective properties were obtained in López-Realpozo et al. (2014) depending only on the unknown $\alpha_{3} a_{p}^{j}$ related to the solution of the local problems (33)-(36):

$$
\begin{aligned}
& p_{11}-i p_{21}=p_{1}\left[1-\frac{V_{2}}{\pi R} \sum_{j=0}^{n-1}{ }_{13} \bar{a}_{1}^{j+1}\left(\theta_{j+1}-\theta_{j}\right)\right] \\
& -\frac{p_{1} V_{2}}{\pi R} \sum_{j=0}^{n-1}\left\{\begin{array}{l}
\sum_{p=1}^{\infty} * \frac{e^{-\frac{i(p+1)\left(\theta_{j}+\theta_{j+1}\right)}{2}}}{p+1} \operatorname{sen}\left[\frac{(p+1)\left(\theta_{j+1}-\theta_{j}\right)}{2}\right]\left[2{ }_{13} a_{p}^{j+1}-(1-\kappa) \delta_{1 p} R\right] \\
+2 \sum_{p=3}^{\infty} *{ }_{13} \bar{a}_{p}^{j+1} \frac{e^{\frac{i(p-1)\left(\theta_{j+1}+\theta_{j}\right)}{2}}}{p-1} \operatorname{sen}\left[\frac{(p-1)\left(\theta_{j+1}-\theta_{j}\right)}{2}\right]
\end{array}\right\}, \\
& p_{12}-i p_{22}=-p_{1}\left[i+\frac{V_{2}}{\pi R} \sum_{j=0}^{n-1} 23 \bar{a}_{1}^{j+1}\left(\theta_{j+1}-\theta_{j}\right)\right] \\
& -\frac{p_{1} V_{2}}{\pi R} \sum_{j=0}^{n-1}\left\{\begin{array}{c}
\sum_{p=1}^{\infty} * \frac{e^{-\frac{i(p+1)\left(\theta_{j}+\theta_{j+1}\right)}{2}}}{p+1} \operatorname{sen}\left[\frac{(p+1)\left(\theta_{j+1}-\theta_{j}\right)}{2}\right]\left[2{ }_{23} a_{p}^{j+1}+i(1-\kappa) \delta_{1 p} R\right] \\
+2 \sum_{p=3}^{\infty} * 23 \bar{a}_{p}^{j+1} \frac{e^{\frac{i(p-1)\left(\theta_{j}+\theta_{j+1}\right)}{2}}}{p-1} \operatorname{sen}\left[\frac{(p-1)\left(\theta_{j+1}-\theta_{j}\right)}{2}\right]
\end{array}\right\} .
\end{aligned}
$$

The unknown constants $a_{p}^{j}={ }_{\alpha 3} a_{p}^{j}$ are computed as solution of the following infinite system of algebraic equations for each arc:

$$
\bar{a}_{p}^{j}+\beta_{1}^{j} R^{2} H_{1} \delta_{1 p} \bar{a}_{1}^{j}+\beta_{1}^{j} R^{2} H_{2} \delta_{1 p} a_{1}^{j}+\beta_{p}^{j} \sum_{k=1}^{\infty}{ }^{*} w_{k p} a_{k}^{j}=\beta_{1}^{j} \delta_{1 p}\left(\delta_{1 \alpha}-i \delta_{2 \alpha}\right) R,
$$


where $\delta_{k p}$ is the Kronecker delta and $\beta_{p}^{j}=\frac{K_{j}(1-\kappa)+\kappa p}{K_{j}(1+\kappa)+\kappa p}$ and $K_{j}$ is the tangential component of the imperfect parameter for each arc.

In the case where the area of mesophase $S_{I}$ is very thin, very good coincidence exists between the imperfect spring (37) and (38) and three-phase model (26) and (27). A condition between the interface and mesophase parameters is found. Thereby, the volume fraction of the interphase in the three-phase model is $V_{I}=\pi\left(h^{2}+2 h R\right) / V$, where $h$ is the aforementioned thickness of the layer $S_{I}$ (see Fig. 2) and the relationship between the nondimensional debonding parameters $K_{j}$ and the interphase property $\kappa_{1 j}$ can be introduced as in Hashin [6], in the following form:

$$
K_{j}=R_{1} \kappa_{1 j} / h
$$

\section{Numerical algorithm based on a finite element method}

In this part, the numerical method proposed to solve the problem (33)-(36) is based on the finite element method. Even if this technique is quite standard, it is rapidly outlined here. For that purpose, it is first necessary to write the variational formulation of the interface problem (33)-(36). First of all, it is convenient to make the change of variable in each phase

$$
\tilde{u}^{(\gamma)}(\xi)=u^{(\gamma)}(\xi)+\xi_{\alpha},
$$

for $\alpha=1,2, \xi \in S$. Then, $\tilde{u}^{(\gamma)}$ is the solution of the problem

$$
\left\{\begin{array}{l}
\nabla^{2} \tilde{u}^{(\gamma)}=0 \text { in } S, \\
\frac{p_{\gamma}}{p_{1}} \frac{\partial \tilde{u}^{(\gamma)}}{\partial n}=\frac{K_{S}(\theta)}{R} \llbracket \tilde{u} \rrbracket \text { in } \Gamma, \\
\llbracket \frac{p_{\gamma}}{p_{1}} \frac{\partial \tilde{u}^{(\gamma)}}{\partial n} \rrbracket=0 \text { in } \Gamma, \\
\langle\tilde{u}\rangle=\left\langle\xi_{\alpha}\right\rangle \text { in } S, \\
\tilde{u}-\xi_{\alpha} S \text {-periodic. }
\end{array}\right.
$$

Then, choosing a test function $v$, which can be discontinuous along the interface $\Gamma$, multiplying the imperfect contact condition by the jump of this test function and integrating this relation among the interface, one obtains

$$
\int_{\Gamma} \llbracket v \rrbracket \frac{K_{S}(\theta)}{R} \llbracket \tilde{u} \rrbracket \mathrm{d} s-\int_{\Gamma} \llbracket v \rrbracket \frac{p_{\gamma}}{p_{1}} \frac{\partial \tilde{u}^{(\gamma)}}{\partial n} \mathrm{~d} s=0 .
$$

Then, on each sub-domain $S_{\gamma}$, one can write

$$
\int_{S_{\gamma}} \frac{p_{\gamma}}{p_{1}} \nabla v^{(\gamma)} \cdot \nabla \tilde{u}^{(\gamma)} \mathrm{d} \xi+\int_{\Gamma} \frac{p_{\gamma}}{p_{1}} \frac{\partial \tilde{u}^{(\gamma)}}{\partial n} v^{(\gamma)} \mathrm{d} s=0,
$$

and, adding the three last equalities (on $\Gamma, S_{1}$ and $S_{2}$ ), a weak formulation of the problem can be written:

$$
\begin{aligned}
& \int_{S_{\gamma}} \frac{p_{\gamma}}{p_{1}} \nabla \tilde{u} \cdot \nabla v \mathrm{~d} \xi+\int_{\Gamma} \frac{K_{S}(\theta)}{R} \llbracket \tilde{u} \rrbracket \llbracket v \rrbracket \mathrm{d} s=0, \\
& \langle\tilde{u}\rangle=\left\langle\xi_{\alpha}\right\rangle \quad \text { in } S, \quad \tilde{u}-\xi_{\alpha} \text { S-periodic. }
\end{aligned}
$$

Finally, using a standard finite element on each subdomain, and a "flat" finite element on $\Gamma$, which has all its nodes on $\Gamma$, the first ones related to $S_{1}$ and the other ones related to $S_{2}$, it is possible to write a rigidity matrix of this problem that is invertible, with standard error estimates (see, for example, [28,29] for more details).

Due to finite element discretization, the integrals (see formulae (11)-(13) for example) for the computation of the average stress and strains are replaced by sums over all elements contributions. In all the computations below, $P 2$ triangular finite elements (conforming piecewise quadratic Lagrangian finite elements) are used. These elements are characterized by six nodes (one on each vertex and one on the middle of each edge). An example of meshed RVE is provided in Fig. 5 for fiber volume fraction 0.4 and fiber arrangement $\mu=60^{\circ}$. 


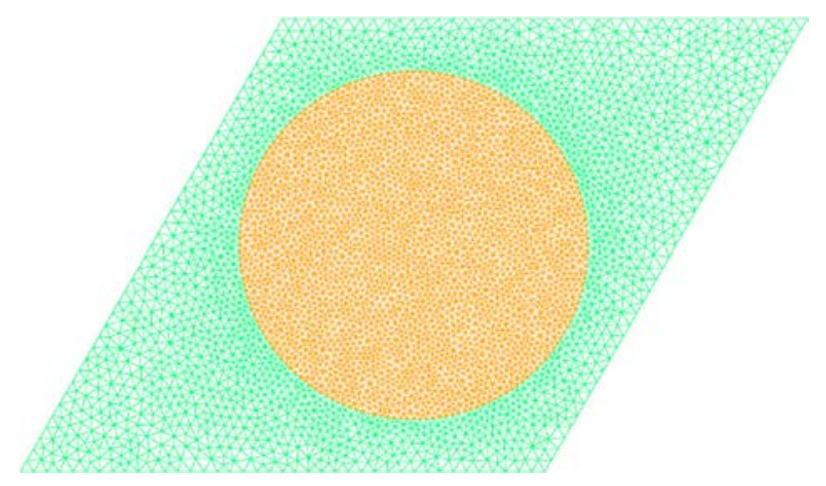

Fig. 5 Meshed RVE for 0.4 fiber volume fraction and $\mu=60^{\circ}$ fiber arrangement

\section{Analysis of results}

In order to illustrate the applicability of the theory developed above, several examples are chosen. As a validation of the model, we study some different particular cases of two-phase composites with hexagonal and square configurations of the periodic cell (Fig. 2). From now on, we shall use the classic short notation for the aforementioned effective elastic constants, i.e., $p_{11}=C_{55}^{*}, p_{12}=C_{54}^{*}, p_{21}=C_{45}^{*}$ and $p_{22}=C_{44}^{*}$. The uniform imperfect elastic spring contact is obtained as a particular case of a stiffness matrix, taking $K_{1}=K_{2}=K_{3}=\cdots K_{j}=K$ with $0<K<\infty$. Now, some comparisons with other theoretical approaches are given in order to validate the present work.

Table 1 presents the normalized effective elastic modulus $C_{44}^{*} / C_{44}^{(1)}$, for perfect contact $\left(K=10^{12}\right)$ computed by the present model using AHM, the Finite Element Method proposed in this work and the results reported in Jiang et al. [20]. The material parameters used for this calculation are $C_{44}^{(2)} / C_{44}^{(1)}=120$ for

Table 1 Effective elastic modulus obtained by Jiang et al. [20], AHM-uniform interface and FEM for composites with perfect contact, square and hexagonal periodic cell and ratio $\left(C_{44}^{(2)} / C_{44}^{(1)}=120\right)$

\begin{tabular}{|c|c|c|c|c|c|c|}
\hline \multirow[t]{3}{*}{$V_{2}$} & \multicolumn{6}{|l|}{$C_{44}^{*} / C_{44}^{(1)}$} \\
\hline & \multicolumn{3}{|l|}{$\mu=60^{\circ}$} & \multicolumn{3}{|l|}{$\mu=90^{\circ}$} \\
\hline & Jiang et al. & AHM & FEM & Jiang et al. & AHM & FEM \\
\hline 0.1 & 1.21815 & 1.21815 & 1,21815 & 1.21816 & 1.21816 & 1,21816 \\
\hline 0.2 & 1.48971 & 1.48971 & 1,48971 & 1.49000 & 1.49000 & 1,49000 \\
\hline 0.3 & 1.83711 & 1.83711 & 1,83711 & 1.83990 & 1.83990 & 1,83990 \\
\hline 0.4 & 2.29764 & 2.29764 & 2,29764 & 2.31343 & 2.31343 & 2,31343 \\
\hline 0.5 & 2.93931 & 2.93931 & 2,93931 & 3.00863 & 3.00863 & 3,00863 \\
\hline 0.6 & 3.90319 & 3.90319 & 3,90319 & 4.18637 & 4.18637 & 4,18638 \\
\hline
\end{tabular}

Table 2 Variations of effective longitudinal shear elastic modulus obtained by Jiang et al. [20], AHM and FEM for a composite with uniform imperfect contact with empty fibers and $C_{44}^{(1)}=30 \mathrm{GPa}$

\begin{tabular}{|c|c|c|c|c|c|c|}
\hline \multirow[t]{3}{*}{$V_{2}$} & \multicolumn{6}{|l|}{$\underline{C_{44}^{*} \equiv C_{55}^{*}}$} \\
\hline & \multicolumn{3}{|c|}{ Hexagonal cell $\left(\mu=60^{\circ}\right)$} & \multicolumn{3}{|c|}{$\underline{\text { Square cell }\left(\mu=90^{\circ}\right)}$} \\
\hline & Jiang et al. & AHM & FEM & Jiang et al. & AHM & FEM \\
\hline 0.1 & 24.54550 & 24.54545 & 24.54546 & 24.54530 & 24.54530 & 24.54531 \\
\hline 0.2 & 20.00000 & 19.99996 & 20.00000 & 19.99590 & 19.99592 & 19.99592 \\
\hline 0.3 & 16.15330 & 16.15326 & 16.15326 & 16.12740 & 16.12740 & 16.12740 \\
\hline 0.4 & 12.85340 & 12.85336 & 12.85336 & 12.76050 & 12.76054 & 12.76054 \\
\hline 0.5 & 9.98430 & 9.98427 & 9.98427 & 9.73960 & 9.73964 & 9.73964 \\
\hline 0.6 & 7.45030 & 7.45029 & 7.45029 & 6.90960 & 6.90954 & 6.90954 \\
\hline
\end{tabular}


hexagonal and square periodic cell. Table 2 shows the behavior of the effective longitudinal shear elastic modulus $C_{44}^{*}=C_{55}^{*}$ for the same arrangement of the periodic cell obtained by Jiang et al. [20], the present model by AHM for void volume fraction $\left(K=10^{-12}\right)$ and the Finite Element Method. The matrix shear modulus used is $C_{44}^{(1)}=30 \mathrm{GPa}$.

In order to show the novelty of this work, we study now the nonuniform imperfect contact for two-phase composites with square cell. We consider the interface of the two-phase composites divided into two pieces $\Gamma=\Gamma_{1} \cup \Gamma_{2}$ and different spring imperfect parameters $K_{1}$ and $K_{2}$, which are assigned constants on $\Gamma_{1}$ and $\Gamma_{2}$, respectively (see Fig. 6), according to the following distribution function $K_{S}(\theta)= \begin{cases}K_{1}, & 0 \leq \theta<\theta_{1}, \\ K_{2}, & \theta_{1} \leq \theta<2 \pi\end{cases}$

Consequently, for illustrating the performance of the considered approach, a composite with hexagonal periodic cell and constituent properties $C_{44}^{(1)}=1, C_{44}^{(2)}=0.0002168$ is taken. For the distribution of the imperfect parameters by arcs, we use $\theta_{1}=\pi / 2$ in the calculations of Table 3 . Two different situations for soft interface are modeled with $K_{1}=10^{5}, K_{2}=0 ; K_{1}=0, K_{2}=10^{5}$ using the AHM (spring and three-phase model) and FEM. Good agreement can be appreciated between the three methods where the relative errors are always smaller than $0.03 \%$.
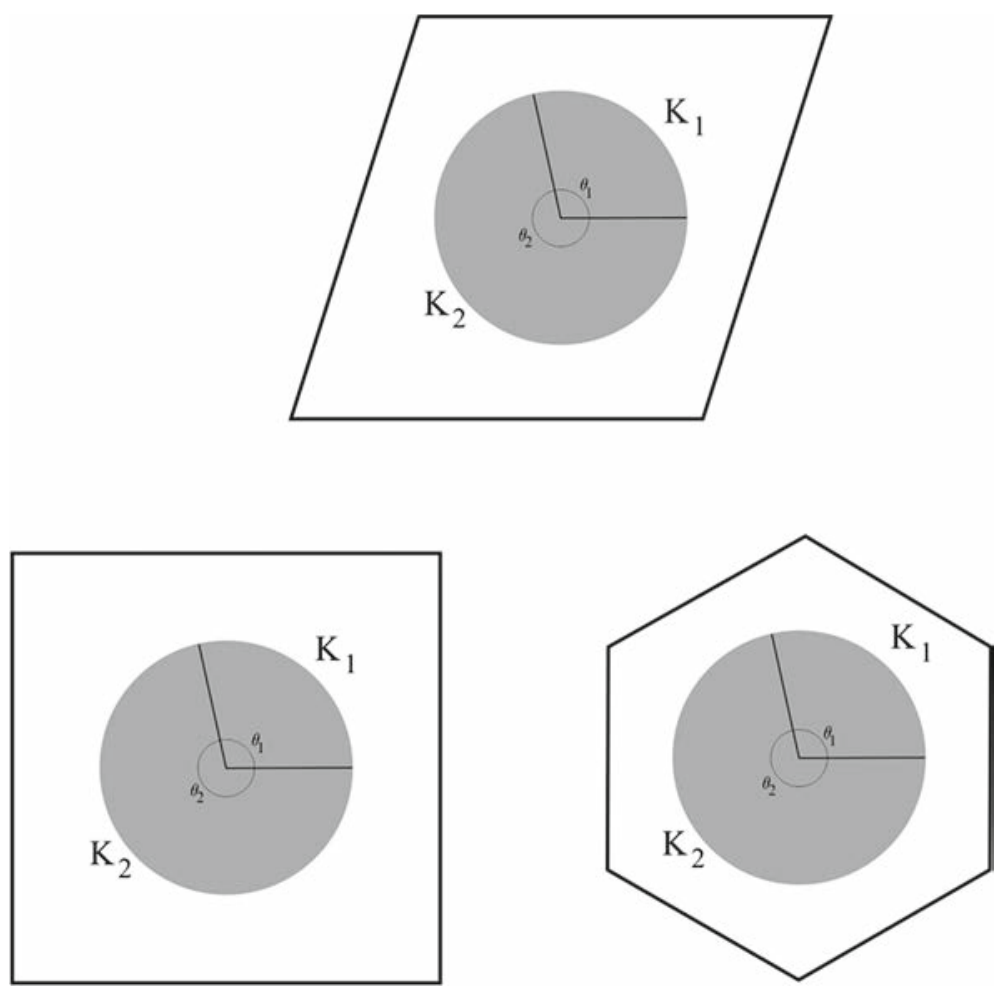

Fig. 6 Two-phase composite with interface divided by two arcs

Table 3 Effective elastic moduli obtained by AHM and FEM approximations for nonuniform imperfect contact and hexagonal periodic cell $\left(\mu=60^{\circ}\right)$. Ratio between the constituent properties is $C_{44}^{(2)} / C_{44}^{(1)}=0.0002168$

\begin{tabular}{|c|c|c|c|c|c|c|c|c|}
\hline \multirow[t]{2}{*}{$V_{2}$} & \multicolumn{4}{|c|}{$C_{44}^{*} / C_{44}^{(1)}\left(K_{1}=10^{5}, K_{2}=0\right)$} & \multicolumn{4}{|c|}{$C_{44}^{*} / C_{44}^{(1)}\left(K_{1}=0, K_{2}=10^{5}\right)$} \\
\hline & $\begin{array}{l}\text { AHM } \\
\text { 3-phase }\end{array}$ & AHM spring & FEM & $\begin{array}{l}\text { Relative error } \\
\text { FEM versus } \\
\text { AHM spring }\end{array}$ & $\begin{array}{l}\text { AHM } \\
\text { 3-phase }\end{array}$ & AHM spring & FEM & $\begin{array}{l}\text { Relative error } \\
\text { FEM versus } \\
\text { AHM spring }\end{array}$ \\
\hline 0.1 & 0.818195 & 0.81819972 & 0.81827746 & 0.0000950 & 0.81823051 & 0.81823555 & 0.81832811 & 0.0000511 \\
\hline 0.2 & 0.666561 & 0.66669543 & 0.6667594 & 0.0000960 & 0.66662112 & 0.66675565 & 0.66684457 & 0.0000565 \\
\hline 0.3 & 0.537619 & 0.5384805 & 0.53853414 & 0.0000996 & 0.53769615 & 0.53855748 & 0.53864309 & 0.0000432 \\
\hline 0.4 & 0.425396 & 0.42848958 & 0.42853803 & 0.000113 & 0.42548651 & 0.42857813 & 0.42866353 & 0.0000929 \\
\hline 0.5 & 0.324705 & 0.33285732 & 0.33289935 & 0.000126 & 0.32480566 & 0.33295393 & 0.33303658 & 0.000162 \\
\hline 0.6 & 0.230374 & 0.24839417 & 0.24842982 & 0.00014 & 0.23048551 & 0.2484966 & 0.24857572 & 0.000264 \\
\hline
\end{tabular}




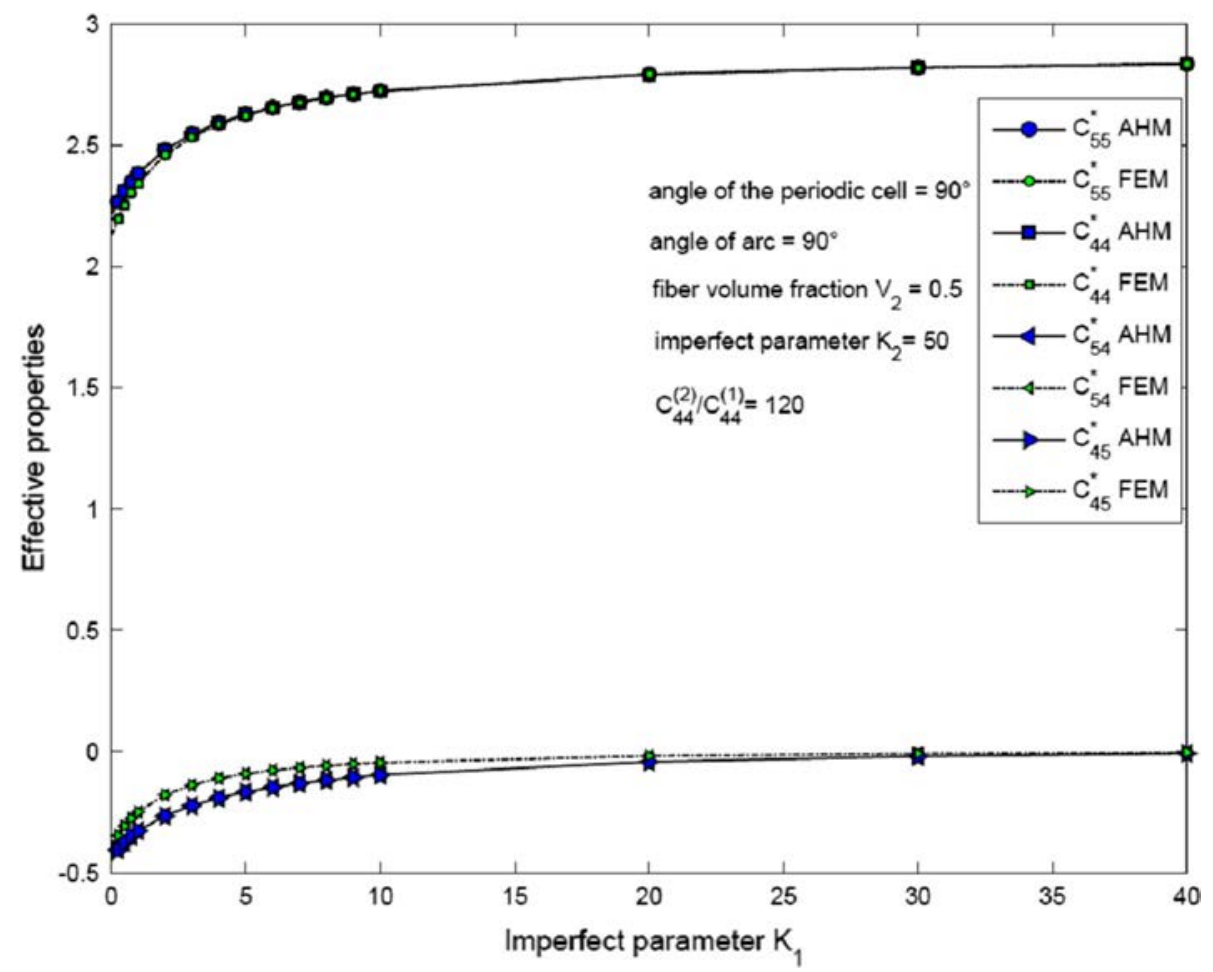

Fig. 7 Comparison of the axial effective properties computed by AHM and FEM where $K_{2}$ is fixed and the other parameter $K_{1}$ changes

Figure 7 shows a comparison between AHM and FEM. The figure describes the behavior of the effective properties $C_{55}^{*}, C_{44}^{*}, C_{54}^{*}$ and $C_{45}^{*}$ computed by AHM and FEM for square cell and a partition of the arc and the nonuniform imperfect parameters given for soft interface modeled by $K(\theta)=K_{1}$ if $0 \leq \theta<\pi / 2, \quad K(\theta)=$ $K_{2}$ if $\pi / 2 \leq \theta<2 \pi$, for $K_{2}=50$, and fiber volume fraction $V_{2}=0.5$. The material parameters used in the computation are $C_{44}^{(2)} / C_{44}^{(1)}=120$. Good agreement between the two models is observed, where the difference is attained for low fiber volume fraction.

Figure 8a, b illustrates a comparison for the overall normalize properties $C_{55}^{*}$ and $C_{45}^{*}$ between three-phase and spring models, both for uniform and for nonuniform interface computed by FEM and AHM approaches. The composite has square periodic cell, and the interface for the nonuniform case is partitioned into two $\operatorname{arcs}$ with length $\pi / 2$ and $3 \pi / 2$, respectively. Hence, the three-phase composite for the nonuniform analysis has the normalized shear interphase property in each element $S_{I 1}$ and $S_{I 2}, \kappa_{11}=p_{I 1} / p_{1}$ and $\kappa_{12}=\kappa_{2}=$ $p_{2} / p_{1}$, respectively. Moreover, the effective properties are considered as a function of $\kappa_{11}$ which varies in the range $\left(0,10^{6}\right)$, whereas $\kappa_{12}=\kappa_{2}=p_{2} / p_{1}$ is constant in the whole range, and the contrast between the fiber and matrix is $\kappa_{2}=120$ (Fig. 8a) and $\kappa_{2}=6$ (Fig. 8b). Consequently, the spring model exhibits a similar pattern and the spring parameters $K_{1}, K_{2}$ assigned to each arc are different. The parameters of imperfection used for the nonuniform analysis (FEM and AHM) are $K_{j}=\kappa_{1 j} p_{1} R_{1} / h$. The composite with uniform interface means that the interphase $S_{I}$ is homogeneous. Accordingly, in the case of the uniform spring model, the imperfect constant is $K_{1}=K_{2}=\kappa_{11} p_{1} R_{1} / h$. The fiber and interphase volume fractions are $V_{2}=0.4$ and $V_{I}=0.001$ in the computation. Notice that the three-phase and spring models coincide in the whole range of the contrast of the stiffness parameter $\kappa_{11}$. Furthermore, when the parameter $\kappa_{11} \rightarrow+\infty$, all models describe the effective properties for a perfect contact of a bi-phase fibrous composite. In the case $\kappa_{11} \rightarrow 0$, the difference between the uniform and nonuniform models is remarkable. In this case, the effective property is weaker in comparison with perfect contact $\left(\kappa_{11} \rightarrow+\infty\right)$ and it is even more weaker when the stiffness $\kappa_{2}=p_{2} / p_{1}$ is low. Hence, the spring uniform model as $\kappa_{11} \rightarrow 0$ reflects a total disbond between the fiber and matrix. These models predict the lower and upper bounds for the effective properties in the range of the $\kappa_{11}$. The two approaches (FEM and AHM) are consistent in the numerical analysis. The differences between them for small value of $\kappa_{11}$ are due to the singularities that appear around the zone where the rigidity of the interface/interphase changes $\left(\theta=0^{\circ}\right.$ and $\theta=90^{\circ}$ ) (these singularities appear for large value of rigidity). It is well known that the global axial behavior 


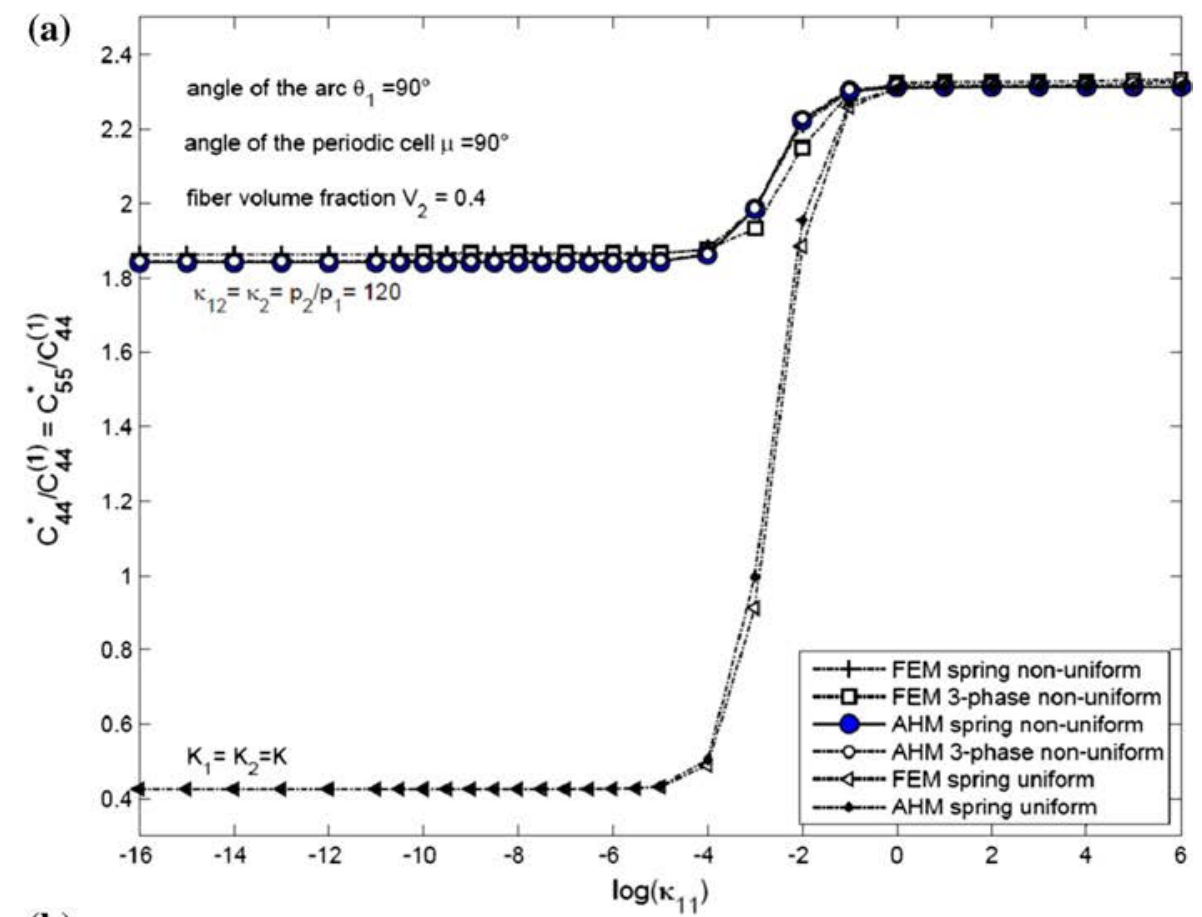

(b)

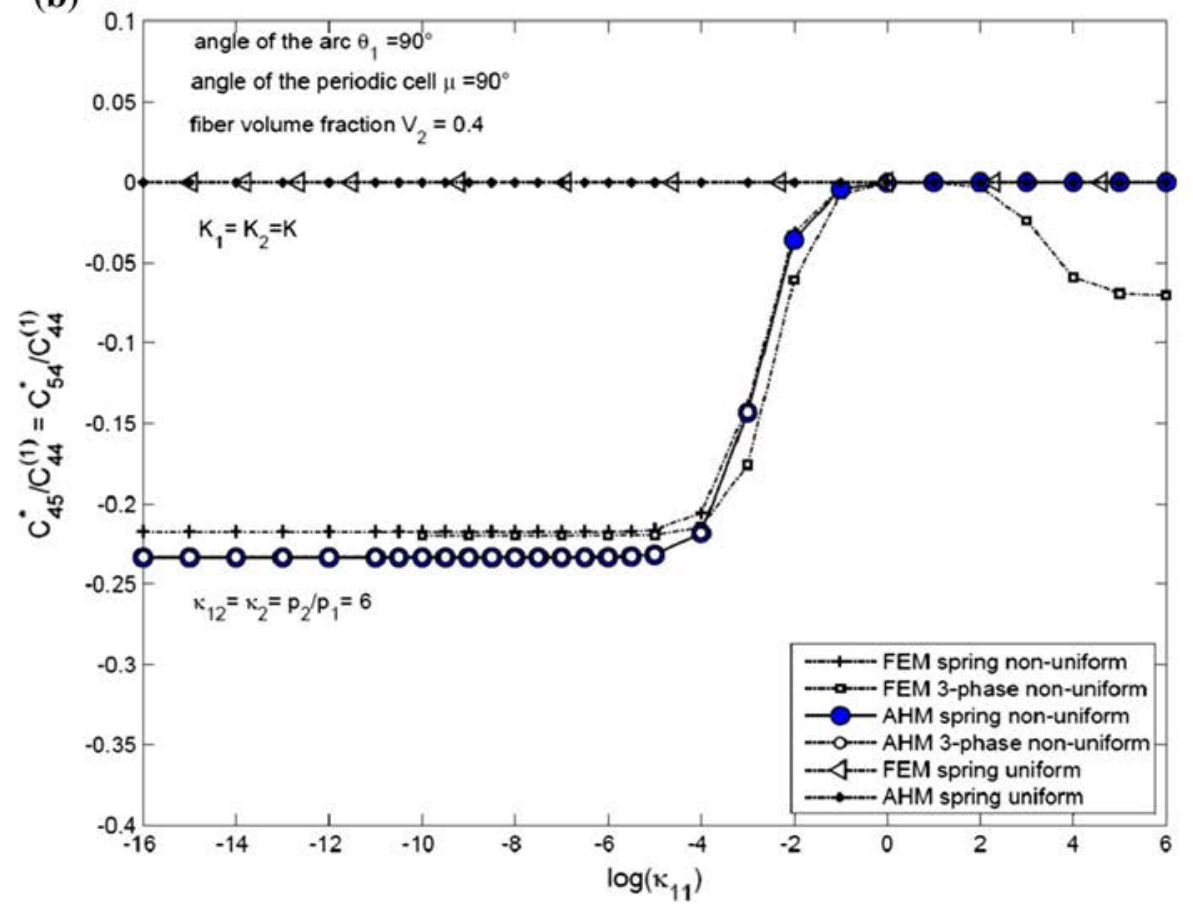

Fig. 8 Different models are compared for the effective properties $C_{55}^{*}$ and $C_{45}^{*}$ in a composite with square periodic cell, uniform/nonuniform imperfect contacts and two different values of contrast between the fiber and matrix $\kappa_{2}=120$ (a) and $\kappa_{2}=6(\mathbf{b})$

in fibrous composites with periodic square cell, isotropic components along the axis $x_{3}$ under perfect contact condition, is transversely isotropic; however, due to the nonuniform imperfection at the interface, the global behavior of this composite changes and it is a monoclinic symmetry so that the effective coefficient $C_{45}^{*}$ is different from zero for the antiplane problem (see Fig. 8b).

A comparison between the three-phase and spring model, both for non-uniform case, is shown in Fig. 9. This figure shows the effective normalized properties $C_{44}^{*}, C_{55}^{*}, C_{45}^{*}=C_{54}^{*}$ varying the length of the arcs of the 


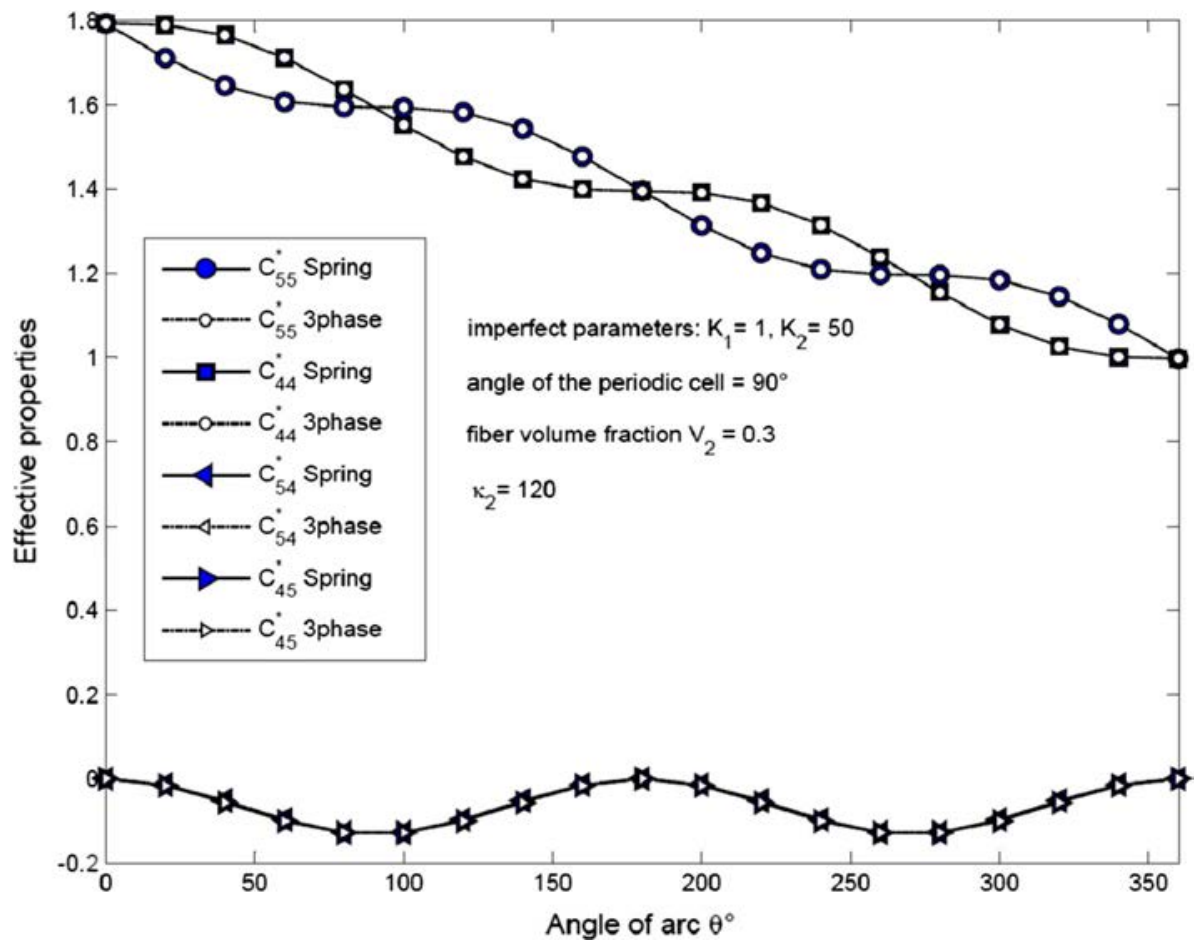

Fig. 9 Behavior of the effective properties by AHM spring and three-phase models for a composite with square cell and nonuniform imperfect interface
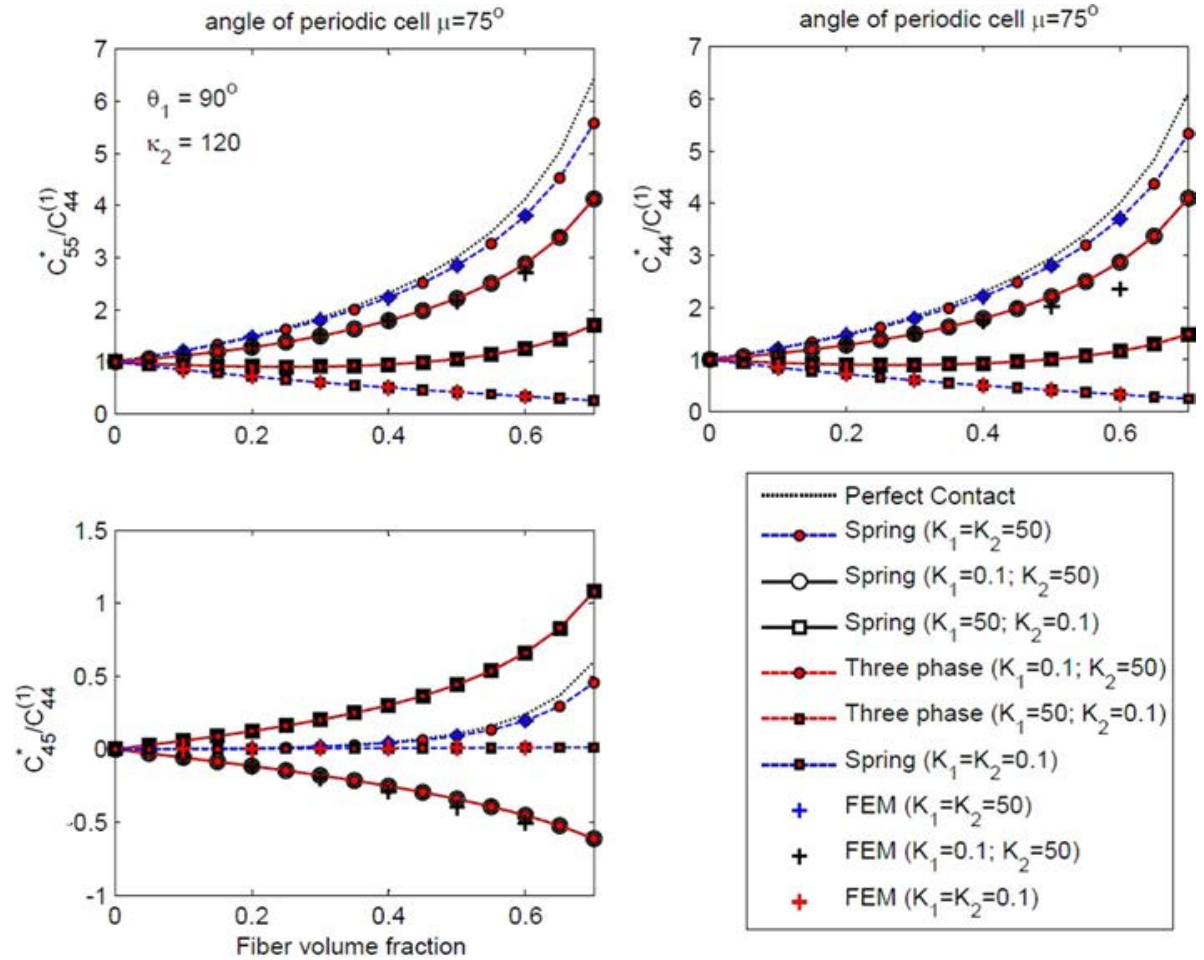

$$
\begin{aligned}
& \text {-........- Perfect Contact } \\
& - \text { Spring }\left(\mathrm{K}_{1}=\mathrm{K}_{2}=50\right) \\
& \rightarrow-\text { Spring }\left(\mathrm{K}_{1}=0.1 ; \mathrm{K}_{2}=50\right) \\
& \rightarrow-\text { Spring }\left(\mathrm{K}_{1}=50 ; \mathrm{K}_{2}=0.1\right) \\
& - \text { Three phase }\left(\mathrm{K}_{1}=0.1 ; \mathrm{K}_{2}=50\right) \\
& + \text { FEM }\left(\mathrm{K}_{1}=\mathrm{K}_{2}=50\right) \\
& +\operatorname{FEM}\left(\mathrm{K}_{1}=0.1 ; \mathrm{K}_{2}=50\right) \\
& +\operatorname{FEM}\left(\mathrm{K}_{1}=\mathrm{K}_{2}=0.1\right)
\end{aligned}
$$

Fig. 10 Effective axial properties of a composite with rhombic cell $\left(\mu=75^{\circ}\right)$ versus the volume fraction using spring and three-phase models via AHM. The influence of the nonuniform interface is considered by different configuration of the imperfect parameters $K_{1}$ and $K_{2}$. The composite exhibits monoclinic symmetry (i.e., $C_{44}^{*} \neq C_{55}^{*}, C_{45}^{*} \neq 0$ and $C_{54}^{*} \neq 0$ ) (color figure online) 
imperfection in the composite where the parameters of imperfections $K_{1}=1, K_{2}=50$ are considered. The material parameters used in the computation are $\kappa_{2}=C_{44}^{(2)} / C_{44}^{(1)}=120$, the fiber volume fraction is $V_{2}=0.3$ and the interphase volume fraction is $V_{I}=0.001$. Notice that the effective normalized properties $C_{44}^{*}$ and $C_{55}^{*}$ are decreasing and different, except when the amplitudes of the arc are $90^{\circ}, 180^{\circ}, 270^{\circ}$ and $360^{\circ}$.

In Fig. 10, the effective axial properties of a composite with rhombic cell versus the volume fraction are studied. The angle of the periodic cell is $\mu=75^{\circ}$, and the contrast between the fiber and matrix used in the calculus is $\kappa_{2}=p_{2} / p_{1}=120$. Some different cases are presented via AHM: first, a composite with perfect contact conditions at the interface, denoted by black dotted line, is considered. The coefficients $C_{44}^{*}$ and $C_{55}^{*}$ possess the biggest rigidity because the fiber is much more rigid than the matrix. When in Fig. 10a, b the imperfect uniform parameter $K=50$ (blue circles and dashed line) is included, the present models indicate that the composite begins to be weaker. The nonuniform interphase considered possesses two arcs of circumference with different length, with angles $\theta_{1}=90^{\circ}, \theta_{2}=360^{\circ}-\theta_{1}$ and the corresponding imperfect parameters $K_{1}$ and $K_{2}$, respectively (see Fig. 6). The coefficients $C_{44}^{*}$ and $C_{55}^{*}$ when $K_{1}<K_{2}$ (black and red circles) are weaker than the uniform imperfection and reciprocally in the case $K_{2}<K_{1}$ (black and red square) the fragility increases. The curve corresponding to the imperfect uniform parameter $K=0.1$ (blue square and dashed line) approximates to the total separation of materials or porous fibers. One remarkable difference of the composite with rhombic periodic cell in comparison with hexagonal or square cell is that it exhibits monoclinic symmetry, i.e., $C_{44}^{*} \neq C_{55}^{*}, C_{45}^{*} \neq 0$ and $C_{54}^{*} \neq 0$. Moreover, in Fig. 10c, d it can be observed that the absolute value of $C_{45}^{*}$ and $C_{54}^{*}$ increases as the nonuniform interphase is considered. The total coincidence between three-phase and spring models can be obtained when the interphase parameter $\eta=h / R_{2}=10^{-5}$ [6]. For major values of $\eta$, some differences appear between the used models, because the thickness $h$ is increased and the mesophase $S_{I}$ has more influence in the properties of the composite. Besides, the validation with FEM indicates the accuracy of the theoretical models calculated by AHM.

\section{Conclusions}

An approach using the asymptotic homogenization method for simulating the nonuniform imperfect bonding in elastic composite materials with oblique angle of the cell is proposed. We introduce different elastic mesophase constants for each partition of the interphase $S_{I}$, between the matrix and inclusions, where a piecewise constant function $p_{I}(\theta)=p_{I j}, \theta_{j}<\theta<\theta_{j+1}$ transmits a load from the matrix to the inclusion. The computation of the analytical expressions (26) and (27) of the shear moduli for three-phase model with nonuniform mesophase, derived by the asymptotic homogenization approach, is realized with good accuracy. The exactitudes of the solutions (26) and (27) were tested with other theoretical models (AHM spring model (37) and (38) and FEM), and good agreement was observed. The nonuniform imperfect interface and the angle of inclination of the cell play an important role in the global behavior of the composite. When the thickness of the interphase is very small $(h \rightarrow 0)$, the three-phase composites behave like two-phase composites. These models can be extended to more complex composites in order to predict the overall properties in the design and manufacturing of one-directional fibrous composite materials.

Acknowledgments The funding of CONACYT project number 129658 and Coordinación de la Investigación Cientifica (COICSTIA-312-14, 313-14) de la UNAM is gratefully acknowledged. The authors gratefully acknowledge to the project SHICHAN, supported by FSP (Cooperation Scientifique Franco-Cubaine) Cuba 2011-26, PROJET No 29935XH and to the project Composite Materials from University of Havana. Thanks to Departamento de Matemáticas y Mecánica, IIMAS-UNAM, for its support and Ramiro Chávez Tovar and Ana Pérez Arteaga for computational assistance.

\section{References}

1. Huang, H., Talreja, R.: Effects of void geometry on elastic properties of unidirectional fiber reinforced composites. Compos. Sci. Technol. 65, 1964-1981 (2005)

2. MansonWakeman, M.D., Bernet, N.: Composite processing and manufacturing: an overview, Compreh Comp. Mater. 2 , 577. Elsevier Science Ltd, Oxford, UK (2000)

3. Kushch, V.I.: Transverse conductivity of unidirectional fibrous composite with interface arc cracks. Int. J. Eng. Sci. 48, 343$356(2010)$

4. Prakash, R.: Significance of defects in the fatigue failure of carbon fibre reinforced. Plast. Fibre Sci. Technol. 14, 171181 (1981)

5. Theocaris, P.S.: The Mesophase Concept in Composites. Springer, Berlin (1987) 
6. Hashin, Z.: Thin interphase/imperfect interface in elasticity with application to coated fiber composites. J. Mech. Phys. Solids 50, 2509-2537 (2002)

7. Guinovart-Díaz, R., Rodríguez-Ramos, R., Bravo-Castillero, J., Sabina, F.J., Maugin, G.A.: Closed-form thermo-elastic moduli of a periodic three-phase fiber-reinforced composite. J. Therm. Stress. 28, 1067-1093 (2005)

8. Abdelmoula, R., Coutris, M., Marigo, J.: Comportement asymptotique d'une interface mince. C. R. Acad. Sci. II 326, $237-$ $242(1998)$

9. Benveniste, Y.: A general interface model for a three-dimensional curved thin anisotropic interphase between two anisotropic media. J. Mech. Phys. Solids 54(4), 708-734 (2006)

10. Klarbring, A.: Derivation of a model of adhesively bonded joints by the asymptotic expansion method. Int. J. Eng. Sci. 29, 493$512(1991)$

11. Rizzoni, R., Lebon, F.: Asymptotic analysis of an elastic thin interphase with mismatch strain. Eur. J. Mech. A Solid 36, 18 (2012)

12. Benveniste, Y., Miloh, T.: Imperfect soft and stiff interfaces in two dimensional elasticity. Mech. Mater. 33, 309-323 (2001)

13. Achenbach, J.D., Zhu, H.: Effect of interphases on micro and macro mechanical behaviour of hexagonal-array fiber composites. J. Appl. Mech. 57, 956-963 (1990)

14. Hashin, Z.: Thermoelastic properties of fiber composites with imperfect interface. Mech. Mater. 8, 333-338 (1990)

15. Hashin, Z.: The spherical inclusion with imperfect interface. J. Appl. Mech. 58, 444-449 (1991)

16. Hashin, Z.: Thermoelastic properties of particulate composites with imperfect interface J. Mech. Phys. Solids 39, 745$762(1991)$

17. López-Realpozo, J.C., Rodríguez-Ramos, R., Guinovart-Díaz, R., Bravo-Castillero, J., Otero, J.A., Sabina, F.J., Lebon, F., Dumont, S., Sevostianov, I.: Effective elastic shear stiffness of a periodic fibrous composite with non-uniform imperfect contact between the matrix and the fibers. Int. J. Solids Struct. 51(6), 1253-1262 (2014)

18. Molkov, B.A., Pobedria, B.E.: Effective characteristic of fibrous unidirectional composite with periodic structure. Mech. Solids. 2, 119-129 (1985) (in Russian)

19. Abolfathi, N., Abhay, N., Ghodrat, K., Chad, U.: A micromechanical characterization of angular bidirectional fibrous composites. Comput. Mater. Sci. 43, 1193-1206 (2008)

20. Jiang, C.P., Xu, Y.L., Cheung, Y.K., Lo, S.H.: A rigorous analytical method for doubly periodic cylindrical inclusions under longitudinal shear and its application. Mech. Mater. 36, 225-237 (2004)

21. Rodríguez-Ramos, R., Yan, P., López-Realpozo, J.C., Guinovart-Díaz, R., Bravo-Castillero, J., Sabina, F.J., Jiang, C.P.: Two analytical models for the study of periodic fibrous elastic composite with different unit cells. Compos. Struct. 93, 709 $714(2011)$

22. Guinovart-Díaz, R., López-Realpozo, J.C., Rodríguez-Ramos, R., Bravo-Castillero, J., Ramírez, M., Camacho-Montes, H., Sabina, F.J.: Influence of parallelogram cells in the axial behaviour of fibrous composite. Int. J. Eng. Sci. 49, 75-84 (2011)

23. López-Realpozo, J.C., Rodríguez-Ramos, R., Guinovart-Díaz, R., Bravo-Castillero, J., Sabina, F.J.: Transport properties in fibrous elastic rhombic composite with imperfect contact condition. Int. J. Mech. Sci. 53, 98-107 (2011)

24. Pobedrya, B.E.: Mechanics of composite materials. Moscow State University Press, Moscow (1984) (in Russian).

25. Bakhvalov, N., Panasenko, G.: Homogenisation: Averaging Processes in Periodic Media. Kluwer, Dordrecht (1989)

26. Muskhelishvili, N.I.: Some Basic Problems in the Mathematical Theory of Elasticity. Noordhoff, Groningen (1953)

27. Shodja, H.M, Tabatabaei, S.M, Kamali, M.T.: A piezoelectric medium containing a cylindrical inhomogeneity: Role of electric capacitors and mechanical imperfections. Int. J. Solids Struct. 44, 6361-6381 (2007)

28. Nairn, J.A.: Numerical implementation of imperfect interfaces. Comput. Mater. Sci. 40, 525-536 (2007)

29. Dumont, S., Lebon, F., Rizzoni, R.: An asymptotic approach to the adhesion of thin stiff films. Mech. Res. Commun. 58, 24 35 (2014) 